\title{
Evolution of Posttraumatic Stress Symptoms and Quality of Life throughout a Cognitive Behavioral Treatment for Workplace Trauma Victims
}

\author{
Lacerte Sophie ${ }^{1,2 *}$, Beaulieu-Prévost Dominic ${ }^{1,3}$, Guay Stéphane ${ }^{1,4}$, Belleville Geneviève ${ }^{1,5}$ and Marchand André Men $^{1,2}$ \\ ${ }^{1}$ Trauma Studies Centre, Institut Universitaire en Santé Mentale De Montréal, Canada \\ ${ }^{2}$ Department of Psychology, Université du Québec à Montréal, Canada \\ ${ }^{3}$ Department of Sexology, Université du Québec à Montréal, Canada \\ ${ }^{4}$ Department of Criminology, Université de Montréal, Canada \\ ${ }^{5}$ Department of Psychology, Université Laval, Canada
}

\begin{abstract}
Background: Posttraumatic stress disorder (PTSD) affects the quality of life of employees exposed to a workplace trauma. Cognitive behavioral therapy (CBT) is an effective treatment for PTSD. Less is known, however, about the effect of CBT for work-related PTSD on symptom reduction and QoL enhancement, and how those variables evolve throughout the therapy.
\end{abstract}

Aim: This study aims to thoroughly monitor the evolution of symptoms and quality of life during and after CBT for work-related PTSD. METHODS: Forty-four participants completed structured interviews and questionnaires four times over the course of treatment (at pre-treatment, after the third session, after the ninth session, and posttreatment) and at follow-up (six months). The assessment of quality of life includes the level of current functioning, general quality of life, and work-related quality of life. CBT included 20 weekly individual sessions.

Results: Our study showed that all outcomes improved significantly at post-treatment and improvements remained stable over the following six months. $69 \%$ of the participants no longer met the criteria for PTSD diagnosis, and half of the sample was able to return to work following psychotherapy. PTSD and QoL significantly improved following each CBT strategy until the end of therapy, and the variables appeared to change synchronously throughout the therapeutic process (i.e., no lagged effect).

Conclusions: The findings suggest that work-related trauma victims can benefit from CBT for PTSD. The addition of specific strategies targeting quality of life in CBT for PTSD seems to be not essential for quality of life improvements, but clinicians should consider adding interventions focused on returning to work.

Keywords: Cognitive behavioral therapy; CBT; Posttraumatic stress disorder; Work-related PTSD; Quality of life; Therapeutic evolution

Abbreviations: ACF: Assessment of Current Functioning; CAPS: Clinician-Administered PTSD Scale; CBT: Cognitive Behavioral Therapy; IRRT: Imagery Rescripting and Reprocessing Therapy; PTSD: Posttraumatic Stress Disorder; QoL: Quality of Life; SCID: Structured Clinical Interview for DSM Disorders; WHOQOL: World Health Organization-Quality of Life; WHOQOL-BREF: World Health Organization-Quality of Life-Brief Version

\section{Introduction}

Posttraumatic stress disorder (PTSD) is the most frequent stress reaction after experiencing a traumatic event (American Psychiatric Association, 2013). Cognitive behavioral therapy (CBT) has been shown to be effective in the treatment of PTSD is recommended by the International Society for Traumatic Stress Studies [1-10]. The efficacy of CBT for PTSD has frequently been demonstrated by the significant reduction of PTSD symptom severity. In the last two decades, several PTSD studies have included a measure as well of QoL as a treatment outcome [11-15]. A recent meta-analysis stated that CBT has a moderately strong effect on QoL for those with anxiety disorders [15]. However, general conclusions on the effect of treatment on QoL are limited, given the methodological flaws and heterogeneity in QoL assessment. Additionally, most studies do not provide a clear definition of QoL [16-18]. The World Health Organization has developed an operationalized definition of QoL: "individuals" perception of their position in life in the context of the culture and value systems in which they live and in relation to their goals, expectations, standards and concerns" (WHOQOL Group) [19-34]. Therefore, the present study will use WHOQOL's definition and will add that QoL is also influenced by the subjective perception of one's level of achieved activity (i.e., functioning) in a specific domain of life. Role functioning appears to be an appropriate QoL measure for individuals in industrialized societies in which performance is highly valued. Furthermore, most studies have used a pre-post design to evaluate the efficacy of treatment on outcomes, showing that QoL improves after symptomfocused psychotherapy. However, this approach does not allow clinical researchers to examine the evolution of outcomes throughout each step of a multicomponent therapy such as CBT. To our knowledge, no study has yet examined the evolution of QoL with each CBT strategy introduced to participants suffering from PTSD. Using a temporal design with closely spaced observations allows researchers to identify

*Corresponding author: Lacerte S, Trauma Studies Centre, Institut Universitaire en Santé Mentale de Montréal 7331 Hochelaga, Montréal (Québec), Canada, H1N 3V2, Tel: 514-251-4000 \#3574; Fax: 514-251-4014; E-mail: sophielacerte@hotmail.com

Received April 29, 2017; Accepted May 05, 2017; Published May 15, 2017

Citation: Sophie L, Dominic BP, Stéphane G, Geneviève B, André M (2017) Evolution of Posttraumatic Stress Symptoms and Quality of Life throughout a Cognitive Behavioral Treatment for Workplace Trauma Victims. J Ment Disord Treat 3: 137. doi:10.4172/2471-271X.1000137

Copyright: $\odot 2017$ Sophie L, et al. This is an open-access article distributed under the terms of the Creative Commons Attribution License, which permits unrestricted use, distribution, and reproduction in any medium, provided the original author and source are credited. 
more complex and precise processes of change, which can help clinicians and patients to understand how evolve outcomes throughout each step of treatment and thus, have more precise expectations of the evolution of the therapy.

Moreover, including quality of life as an outcome in studies of PTSD symptom-focused treatment reflects the assumption that reduced quality of life is secondary to symptoms. Immediate improvement in symptoms may take time to influence one's perception of satisfaction in life and his functioning. For example, decrease in anger and irritability might motivate a person to get closer with the spouse, but time is usually needed to rebuild a close relationship. Few studies have tried to examine the lagged effect between PTSD and QoL in the course of therapy. Mixed results are revealed in the literature: some studies of Schnurr et al. [29] have demonstrated that improvements in symptoms are associated with changes in QoL whereas others showed evidence of successive improvements (i.e., a change in QoL is followed by improvements in PTSD and, subsequently, a change in functioning).

This study examines the evolution of PTSD and QoL throughout CBT for employees who experienced workplace trauma. Approximately $1.6 \%$ of workers suffer from PTSD following a traumatic event experienced in the workplace or during off-site work of Muschalla and Linden [24]. Violence and assault appear to be the most frequently experienced types of workplace trauma, as by Lacerte et al. [18] and compared to other types of events, these traumas generate higher severity PTSD and possibly worsen QoL, by Sijbrandij et al. [31]. Workers with PTSD may absent themselves from work, which can affects significantly the QoL of the employee, considering that work is a source of self-satisfaction and socialization. Thus, the rehabilitation of mentally injured workers appears essential to enable employees to return to work. A recent review noted the lack of research on interventions for work-related PTSD [32].

Thus, the current paper aims to examine the evolution of symptoms and QoL throughout CBT for PTSD in individuals exposed to workplace trauma. To fully capture the experience of patients, three aspects of QoL will be examined (general QoL, level of current functioning, and work-related QoL). We hypothesize that symptoms and QoL will significantly improve after CBT for PTSD. Moreover, we aim to monitor the evolution of outcomes with each CBT strategy being introduced and explore the relation between changes in PTSD symptoms and changes in QoL throughout therapy.

\section{Method}

\section{Participants}

Participants were recruited through referrals to the Trauma Studies Centre of the institute, located in Montreal (Canada). The participants were included if (a) they were between the ages of 18 and 65 years, (b) they have been exposed to a traumatic event at work, and (c) they were given a primary diagnosis of PTSD based on SCID assessment. The following exclusion criteria were used: (a) experience of repeated war-related traumas, (b) alcohol or substance abuse/dependence, (c) active suicidal ideation, (d) past or present psychotic episode(s), (e) bipolar disorder, (f) mental retardation; (g) on-going threat (e.g., continued harassment) and (h) overwhelming personal problems (e.g., involvement in a trial).

The participants were required to consent to not starting pharmacological treatment or any other psychological treatment during the study. If the participants were already using a pharmacological treatment, we asked them not to modify their dosage during the course of the psychotherapy if possible. All participants read and signed a consent form explaining the research project before participation. No financial compensation was offered. The ethics committee of the Institut Universitaire en Santé Mentale de Montréal approved the research project (\# 2008-024). Of the 44 participants who were accepted to participate in our study, 40 of them completed the treatment, and 36 participants were reached at 6-month follow-up (Figure 1).

\section{Measure}

\section{PTSD and comorbid mental health disorders}

The Structured Clinical Interview for DSM-IV-TR Axis I Disorders (SCID-I; First, Spitzer, Gibbon, and Williams, 1996) was used to assess PTSD diagnosis and other Axis I disorders (e.g., major depression). PTSD severity was evaluated by the Clinician-Administered PTSD

Flow chart

217 participants attended face-to-face initial evaluation for eligibility

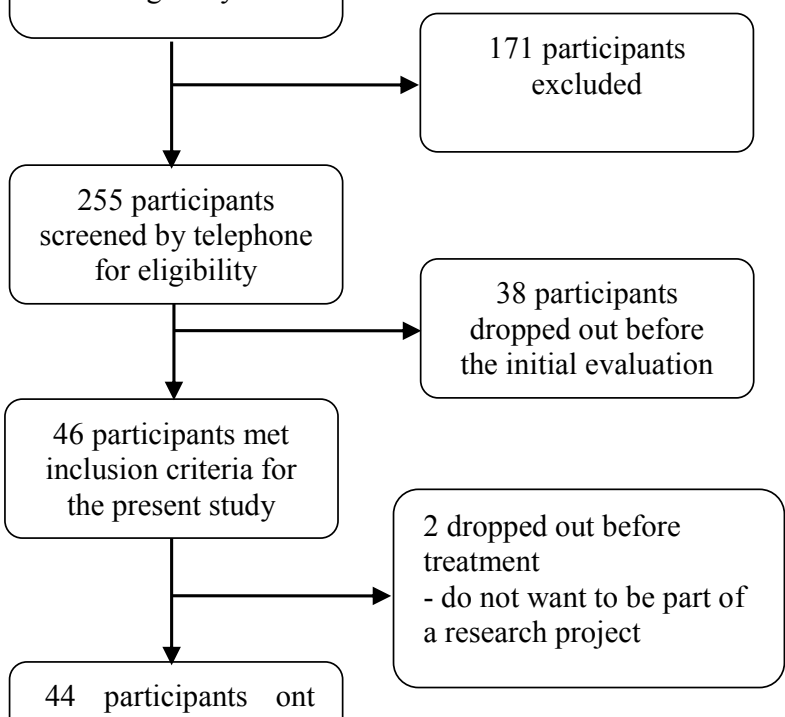
complété la thérapie

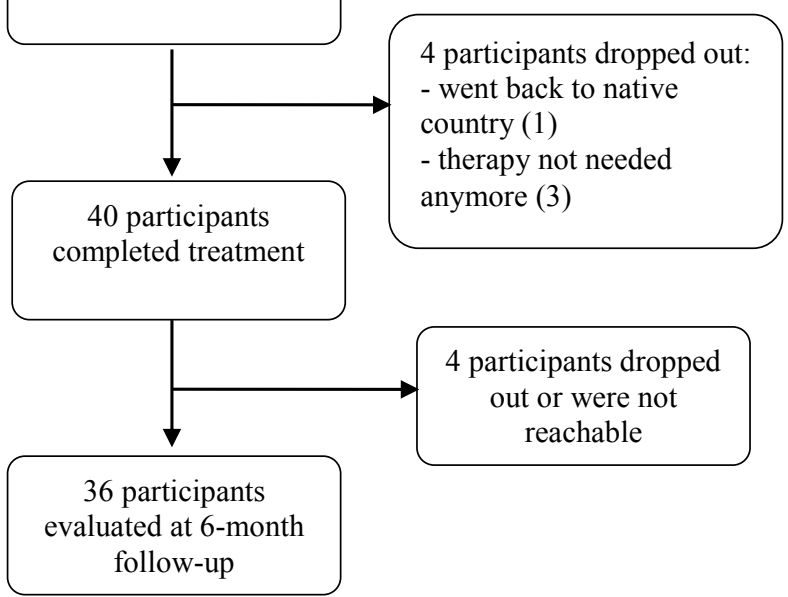

Figure 1: Flow chart of psychological treatment during the study. 
Scale [4]. The frequency and intensity of each PTSD symptom were assessed using a five-point Likert scale on which higher scores reveal more severe PTSD symptoms.

\section{General QoL}

The World Health Organization Quality of Life - BREF (WHOQOLBREF; WHOQOL Group, 1998) is a self-report questionnaire widely used to assess general QoL. Four domains of QoL are evaluated with 24 items rated on a five-point Likert scale, yielding four subtotals. For the purpose of the present study, a total score was also calculated by computing the mean of all domain scores. The WHOQOL-BREF subscale intercorrelations were moderate to large (Pearson's $r=0.40$ to $\mathrm{r}=0.66, \mathrm{p}<0.01)$, and good internal consistency was confirmed $(\alpha=0.82)$ [7]. Lower scores indicate poor QoL.

\section{Level of functioning}

The current level of functioning was measured with the Assessment of Current Functioning (ACF), a questionnaire adapted by the main researchers (D.B.P. and A.M.) from Freeston's scale. In a clinical interview, the participants were asked to what extent the presence of PTSD symptoms influences their functioning in six life spheres: work, marital relations, family life, social life, leisure/holidays, and daily activities. Based on the participant's answers, the evaluators rated their level of functioning on a Likert scale ranging from 1 (no difficulties) to 9 (severe difficulties). Good internal consistency was confirmed for this study $(\alpha=.86)[7]$.

\section{Work-related QoL}

Based on Gladis, Gosch, Dishuk, and Crits-Christoph's [13] recommendations for QoL assessment, work-related QoL includes three components: employment status (i.e., an indication of sociomaterial condition), satisfaction and functioning at work. Because the study was not initially intended to assess work-related outcomes, we decided to separately analyse one item in the WHOQOL-BREF ("How are you satisfied with your capacities for work?") and one item in the ACF questionnaire ("To what extent does the presence of PTSD influence functioning at work?"). Those items were not considered in the total score for the corresponding questionnaire. Employment status was identified in terms of whether the participants reported working or not working at the time of assessment.

\section{Treatment and procedure}

The intervention protocol was adapted from an empirically validated CBT treatment for PTSD. The length of the therapy was increased to 20 sessions, giving enough time to address persistent difficulties (e.g., persistent avoidance behaviors). Eight psychologists performed the 20 weekly individual 90 -min sessions. The psychologists were all members of the professional order of Quebec. They were all experts in CBT for PTSD, and they were trained and supervised in applying the manualized treatment for our project by two psychologists who were also authors (A.M. and S.G.). The participants received a manual describing symptoms and therapy, and complete homework. The treatment was composed of three phases: (a) a psychoeducative intervention regarding PTSD symptoms and the teaching of anxiety management strategies (e.g., diaphragmatic breathing) (sessions 1 to 3); (b) exposure to memories of the traumatic event and cognitive restructuring if needed (sessions 4 to 9); and (c) in vivo exposure to trauma-related stimuli and to avoided situations, with cognitive restructuring if necessary (sessions 10-19) and relapse prevention (session 20). The participants were interviewed five times throughout the course of therapy, delimitating each CBT step: before therapy (T0), after the first three sessions (T1), at the ninth session (T2), at the end of therapy(T3), and 6 months after therapy (T4). These assessments were delivered by one of the seven graduate students in clinical psychology trained to perform the clinical interviews for this project and supervised by two of the authors (A.M. and S.G.). Each evaluation and each session were audio-recorded to verify that the evaluators and psychologists correctly implemented the protocol. Inter-rater agreement on the assessment of the PTSD diagnosis was computed using independent experts who were blind to the study's hypotheses to code the audio-recorded session. The experts listened to one-third of the assessments. In sum, the judges agreed on all (100\%) evaluations at pre-test and agreed on $91.3 \%$ of the evaluations at post-test. Treatment integrity was calculated for $20 \%$ of all completers' treatment sessions and was found excellent (99.62\% agreement).

\section{Data analyses}

SPSS (19th edition) was used. Normality hypotheses were verified for all variables. A ceiling effect on the work functioning variable was observed. Consequently, we dichotomized it by assigning a value of "0" to participants with moderate to high levels of functioning and a value of "1" for low levels of functioning. Fisher's test and Student's $t$ test revealed no difference between the participants who completed the treatment and those who did not with respect to any of the demographic or outcome variables at baseline. Therefore, only the completers' sample was considered in the study analyses. Moreover, preliminary analyses revealed equivalent results when using either intentionto-treat analyses or only data from participants who completed the study. Therefore, the aforementioned analyses were completed in a more conservative manner (i.e., excluding intention-to-treat data). Thus, only the participants who completed the follow-up $(n=36)$ were considered in the longitudinal analyses.

Repeated-measures analyses of variance were performed on the continuous outcome variables to detect therapeutic changes. When the sphericity hypothesis was violated, a Greenhouse-Greiser correction was considered. Repeated contrasts were used for pairwise comparisons of time assessments. Cochran's Q tests were used to detect differences in the dichotomous variables from baseline to follow-up. Pairwise comparisons were then computed using McNemar's tests. For the second objective of this study, a change score was computed for each outcome by subtracting the score at one time point from the score at the following time point. The relationships between changes in PTSD and QoL variables were then identified by Pearson's correlations. The threshold of statistical significance was set at $\mathrm{p}<0.05$ for all analyses.

\section{Results}

\section{Sample characteristics}

The characteristics of the sample are shown in Table 1 . The participants worked in the following areas: administration and management $(n=4)$; sales and service $(n=13)$; health and social work $(n=11)$; emergency and protective service $(n=8)$; and trades, transport and manufacturing $(\mathrm{n}=8)$. They have been victims of work accident (e.g., hand injury in manufacturing), assaulted physically or verbally by clients or colleagues, or have witness the injury or death of someone else's (e.g., police officer in a crime scene). Twenty-five participants (57\%) were taking medication for PTSD or related problems (e.g., depression), such as antidepressants, benzodiazepines, and atypical antipsychotics. No statistical differences were found between participants who were taking medication for PTSD and those who did not on all outcomes, $\mathrm{p}>0.05$. (Table 1 ) 
Citation: Sophie L, Dominic BP, Stéphane G, Geneviève B, André M (2017) Evolution of Posttraumatic Stress Symptoms and Quality of Life throughout a Cognitive Behavioral Treatment for Workplace Trauma Victims. J Ment Disord Treat 3: 137. doi:10.4172/2471-271X.1000137

Page 4 of 7

\section{The evolution of symptoms and QoL}

Repeated-measures analyses of variance showed statistically significant effects of time on PTSD severity, current functioning and general QoL (Table 2). Each QoL domain of the WHOQOL-BREF improved statistically over the course of treatment. The effect sizes (eta squared) for all time effects were moderate to large ( $\eta 2=0.31$ to 0.58 ). At post-test, $72 \%$ of the sample no longer met the criteria for PTSD. No statistically significant changes from post-treatment to 6-month follow-up were found for any of the outcomes; thus, treatmentrelated improvements appeared to remain stable in the following months (Figure 2). With regard to work-related QoL specifically, the participants' self-reported functioning at work and work satisfaction statistically improved from pre- to post-treatment and remained stable after therapy (Table 2). Of the participants who were not working at intake $(n=31)$, nine mentioned working at post-test and six others mentioned working in the following six months (Table 2 and Figure 2).

\begin{tabular}{|c|c|c|}
\hline Characteristics & M (SD) & n (\%) \\
\hline Age & $40.86(12.20)$ & \\
\hline Gender (female) & & $25(57)$ \\
\hline Marital status (in couple) & & $20(45)$ \\
\hline \multicolumn{3}{|l|}{ Work status } \\
\hline On medical leave or not & & $39(89)$ \\
\hline Working Disability compensation & & $34(94)$ \\
\hline \multicolumn{3}{|l|}{ Education } \\
\hline Secondary school & & $15(34)$ \\
\hline College and technical & & $23(52)$ \\
\hline Training University & $13.46(20.34)$ & $6(14)$ \\
\hline \multicolumn{3}{|l|}{ Number of months since trauma } \\
\hline Types of workplace trauma & & $9(20)$ \\
\hline Accident/disaster & & $27(61)$ \\
\hline Victim of violence & & $8(18)$ \\
\hline Witness injury/death & 79.05 (14.35) & \\
\hline \multicolumn{3}{|l|}{ CAPS severity } \\
\hline Comorbid mental disorders & & $20(46)$ \\
\hline Anxiety disorders ${ }^{a}$ & & $24(55)$ \\
\hline Mood & & $11(25)$ \\
\hline Disorders & & None \\
\hline
\end{tabular}

Table 1: Sample characteristics at baseline $(n=44)$.

\section{Improvements in outcomes across CBT strategies}

Pairwise comparisons revealed statistically significant improvements at each step of therapy (i.e., from T0 to T3) for PTSD. Same results are observed for QoL outcomes, except for current functioning, for which the participants did not statistically improve from pre-treatment (T0) to the assessment after the third session (T1). Relationship between changes in outcome, (Figure 3), we graphically displayed the correlations between changes in PTSD severity and QoL outcomes (including general QoL, functioning, and work-related QoL) at different time points during therapy (from T0 to T3). The results revealed statistically significant correlations at each step of therapy between PTSD severity and general QoL and between PTSD severity and the level of current functioning. However, no association was found between those variables at the first therapeutic step (corresponding to psychoeducation and anxiety management). Thus, those outcomes appeared to improve synchronously throughout most of the therapeutic process. From T0 to T1, only changes in employment status were associated with changes in posttraumatic stress symptoms. From T1 to T3, improvements in self-reported work satisfaction and work functioning were statistically associated with changes in PTSD symptoms. No lagged effects (i.e., associations between changes in one outcome at one time interval and changes in another outcome at a subsequent time interval) were detected ( $>0.05$ ) (Figure 3).

\section{Discussions and Conclusion}

Our study reveals several findings. First, all outcomes (PTSD severity, general QoL, level of current functioning and work-related QoL) improved significantly after the CBT for PTSD.

Therapeutic gains were maintained six months later. Even of our study did not include a control group (i.e., without treatment) to control for effects of time or regression to the mean, our results are coherent with earlier findings with veterans like Khoo, Dent, and Oei, Schnurr and Lunney. However, our rate of improvement in employment status from pre- to post-treatment (29\%) appears lower than the average rate of $85 \%$ reported by a recent systematic review of studies evaluating the efficacy of exposure-based treatments for PTSD among work-related injured patients. The fact that our treatment protocol did not include a specific intervention targeting work rehabilitation can partially explain our lower return-to-work rate. Further studies are needed to evaluate treatment-related improvements in occupational outcomes among PTSD workers.

\begin{tabular}{|c|c|c|c|c|c|c|c|}
\hline \multirow[t]{2}{*}{ Variable } & \multirow[t]{2}{*}{ TO } & \multirow[t]{2}{*}{$\mathrm{T} 1$} & \multirow[t]{2}{*}{ T2 } & \multirow[t]{2}{*}{ T3 } & \multirow[t]{2}{*}{ T4 (follow-up) } & \multicolumn{2}{|c|}{ Time effect } \\
\hline & & & & & & $F(d f)$ & $\eta^{2}$ \\
\hline PTSD severity $M(S D)$ & $79.00(14.17)$ & $70.42(19.78)$ & $52.78(29.19)$ & $34.72(27.94)$ & $38.39(30.55)$ & $47.89(2.55)$ & 0.58 \\
\hline Current functioning $M(S D)$ & $5.62(1.46)$ & $5.65(1.83)$ & $4.47(2.56)$ & $3.03(2.12)$ & $3.50(2.37)$ & $32.58(4)$ & 0.48 \\
\hline \multicolumn{8}{|l|}{ General QoL M (SD) } \\
\hline Physical health & $46.03(16.51)$ & $50.31(18.03)$ & $60.60(17.66)$ & $64.70(20.35)$ & $63.06(22.71)$ & $13.12(2.25)$ & 0.32 \\
\hline Psychological health & $38.30(17.11)$ & $43.57(15.27)$ & $51.43(20.59)$ & $57.64(23.52)$ & $57.64(22.43)$ & $19.06(4)$ & 0.40 \\
\hline Social relations & $39.29(16.98)$ & $47.38(19.15)$ & $53.93(21.79)$ & $61.92(21.80)$ & $59.72(22.11)$ & $13.46(4)$ & 0.32 \\
\hline Environment & $56.71(14.92)$ & $60.27(14.29)$ & $66.03(14.97)$ & $67.14(15.45)$ & $66.67(16.34)$ & $12.54(4)$ & 0.31 \\
\hline Total & $44.38(14.32)$ & $50.38(13.44)$ & $58.00(15.68)$ & $62.85(18.19)$ & $61.77(18.56)$ & $21.75(2.33)$ & 0.43 \\
\hline \multicolumn{8}{|l|}{ Work-related QoL } \\
\hline Work satisfaction $M(S D)$ & $1.83(1.02)$ & $2.07(1.08)$ & $2.80(1.06)$ & $3.30(1.26)$ & $3.30(1.24)$ & $24.20(4)$ & 0.46 \\
\hline \multicolumn{8}{|l|}{ Work functioning $n(\%)$} \\
\hline High Employment status $n(\%)$ & $4(11)$ & $7(19)$ & $8(22)$ & $14(39)$ & $20(56)$ & $23.39(4)^{a}$ & $n / a$ \\
\hline Working & $5(14)$ & $5(14)$ & $6(17)$ & $12(33)$ & $18(50)$ & $31.90(4)^{\mathrm{a}}$ & $n / a$ \\
\hline
\end{tabular}

Table 2: The effect of time on PTSD severity, QoL, functioning and occupational outcomes during CBT treatment. 


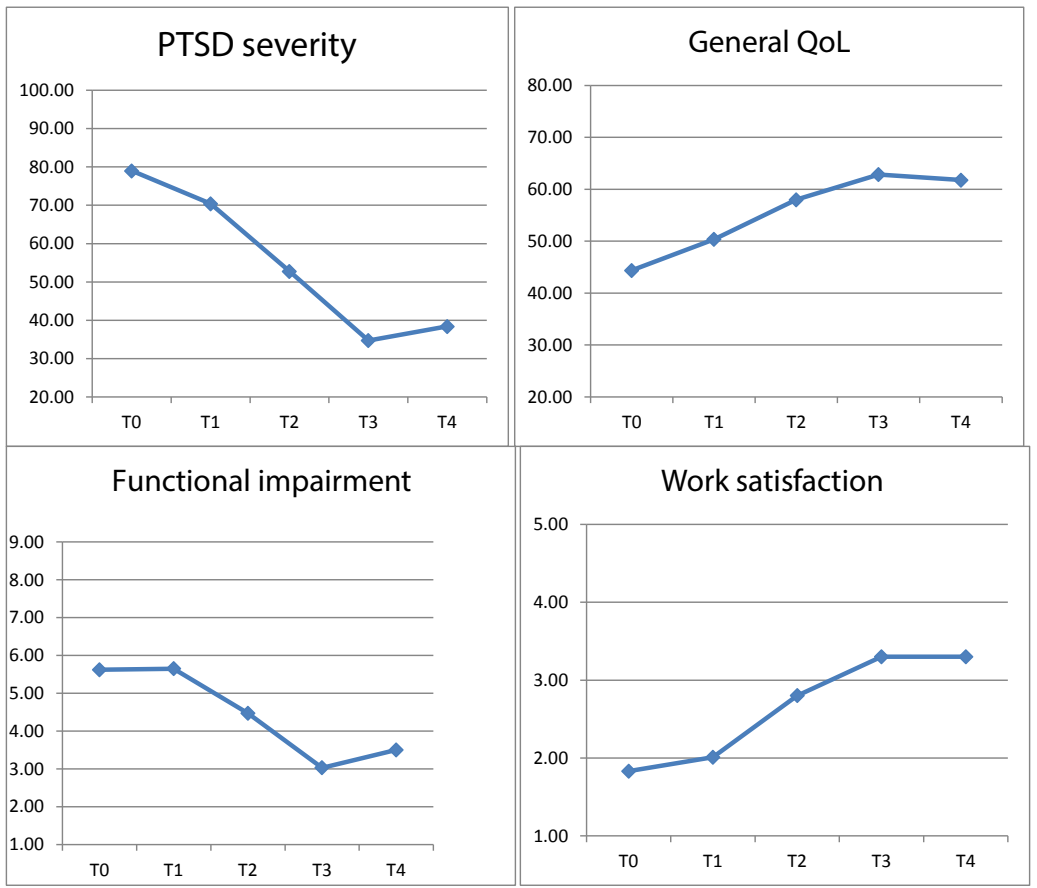

Figure 2: The evolution of treatment outcomes $(n=36)$.

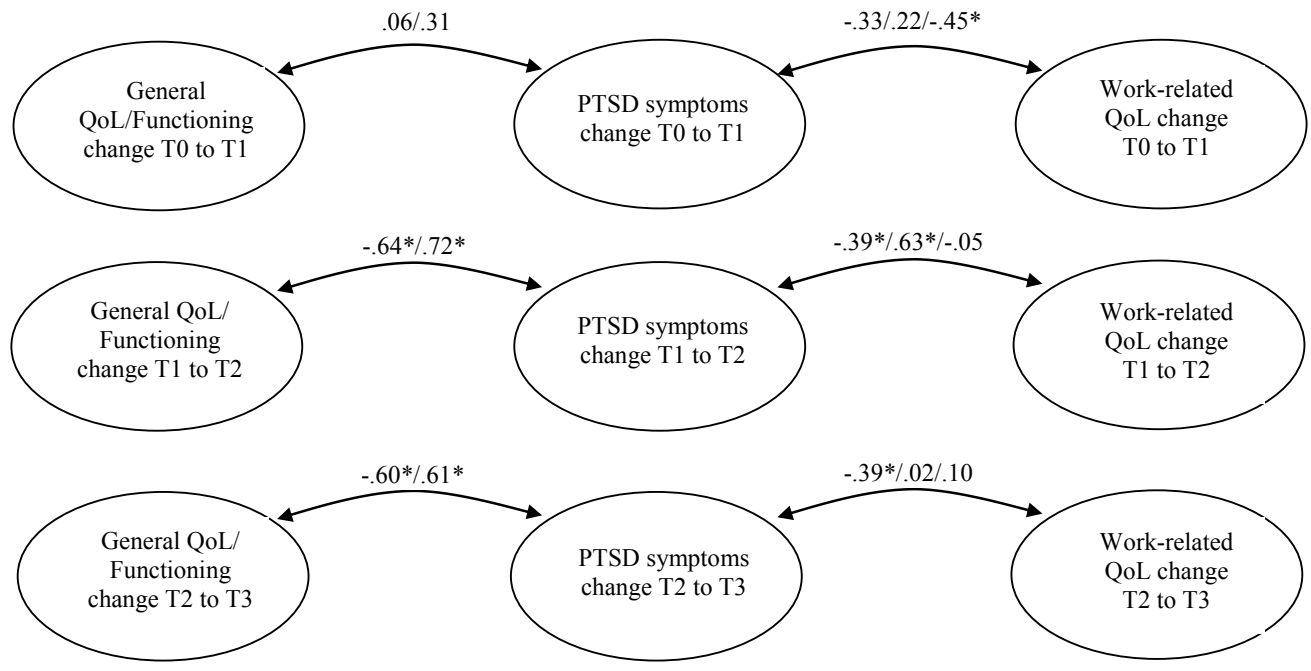

Figure 3: Relationships among changes in PTSD severity, QoL, functioning and employment status $(n=36)$.

Secondly, the results of the present study revealed significant improvements in PTSD and QoL with each CBT strategy introduced (psychoeducation, imaginal exposure and in vivo exposure). Psychoeducation about PTSD can assist patients to understanding their actual state and thus diminish the anxiety associated with not knowing what is happening with their mental and physical health. We believe that psychoeducation may also alter the sufferer's perception of his situation resulting from the presence of PTSD and raise his hopes of recovery, thereby affecting self-perceived QoL. However, this strategy may not be enough to improve the patient's current functioning. Imaginal and in vivo exposures are known for addressing trauma-related anxiety and avoidance behaviors, which are considered core symptoms of PTSD. Diminished anxiety and avoidance behaviors may lead to better general well-being because patients increasingly recognize gains in their mental health recovery. Gradually, patients may feel less incapacitated by the presence of PTSD, gain control of their lives and increase their social and daily activities-all of which can contribute to greater QoL. However, due to the design of our study, we cannot confirm that improvements in symptoms and QoL are due to the specific influence of each CBT component. Results may reflect the additive effects of the strategies, or the effect of time. Dismantling studies with a control group (i.e., without treatment) are needed to address these limitations of interpretation of results.

Thirdly, our study showed that changes in PTSD and QoL outcomes were synchronous throughout therapy. The assumption 
Citation: Sophie L, Dominic BP, Stéphane G, Geneviève B, André M (2017) Evolution of Posttraumatic Stress Symptoms and Quality of Life throughout a Cognitive Behavioral Treatment for Workplace Trauma Victims. J Ment Disord Treat 3: 137. doi:10.4172/2471-271X.1000137

that QoL changes are secondary to PTSD changes (i.e., lagged effect) was not supported. Because the therapy employed strategies targeting specifically PTSD symptoms, it is possible to conclude that there is no need for additional strategies for the enhancement of QoL as changes in PTSD is associated with changes in QoL throughout therapy. Some treatment studies of anxiety disorders have reached the same conclusion. Regarding specific occupational variables, our study also showed in general that changes in employment were not related to changes in PTSD severity. Thus, CBT targeting only PTSD may not be sufficient to influence the returning to work of employees. Standard CBT could be supplemented with strategies focusing specifically on this matter for patients who have experienced a traumatic event in the workplace. For example, Grunert et al. [14] suggest the systematic inclusion of items related to the work environment in graded in vivo exposure exercises. Persistent negative emotions related to the workplace trauma (e.g., anger toward the employer or other employees) also need to be addressed before returning to work. Cognitive restructuring and emotion regulation strategies can be applied. Clinicians can also use imagery rescripting and reprocessing therapy (IRRT) with patients for whom imaginal exposure fails because of strong negative emotions, such as anger or guilt. The goal of this therapy is to alter maladaptive trauma-related schemas (e.g., vulnerability) and replace them with mastery schemas. In fact, IRRT has proven to be a potent intervention. The therapist could also assist the patient in using adequate coping skills when difficulty arises at work (e.g., work tasks, colleagues' reactions). Nonetheless, the cooperation of the employer and of any other recovery agent is paramount to the effectiveness of these interventions.

\section{Limitations and Strengths}

Several limitations can be noted in our study. First, our sample size was somewhat small but sufficient for detecting significant effects with a satisfactory statistical power. Our sample is homogeneous, all participants experienced a traumatic event at work, and thus generalization of results is limited. Because the study was not originally designed to evaluate work-related QoL, we analyzed specific items derived from the questionnaires that were already being administered, which limits the validity and reliability of results obtained for workrelated QoL. Additionally, the correlational analyses do not permit definitive inferences about cause and effect or allow for the identification of the directionality of the results. It is possible that improvements in outcomes after therapy were influenced by the expectations of the therapeutic efficacy; thus, participants could have reported better scores in questionnaires because they felt that this was expected. However, no such expectations were conveyed from the project personnel to the participants. Despite these limitations, this study has several strengths. The study's prospective and repeated-measures design allowed observation of the trajectory of different outcomes during and after treatment. Moreover, treatment and assessment fidelity were excellent. Finally, to fully capture the experience of patients, the treatment outcomes included not only symptoms severity but also QoL. This later variable was examined with three important components, such as general QoL, current functioning and work-related QoL.

\section{Future Research}

Inclusion of a control group (e.g., wait-list group) would be needed to confirm the influence of treatment on outcomes. A dismantling design study would also be useful to separate the influence of each treatment strategy on outcomes. It would be helpful if future studies followed patients for one or two years to support conclusions regarding the maintenance of improvement in therapeutic gains, especially for
QoL outcomes. Moreover, future studies are needed to evaluate treatment-related improvements in occupational outcomes among PTSD workers. We recommend the use of questionnaires specifically addressing QoL at work, such as the Quality of Working Life Systemic Inventory or the Work Limitations Questionnaire. Investigating the factors that contribute to improvements in QoL (general or workrelated QoL) could also be relevant to enhance our understanding of the processes underlying changes in treatment outcomes.

\section{Acknowledgements}

This research was supported by a scholarships awarded by the first author by the Institut de Recherche Robert-Sauvé en Santé et en Sécurité du travail and by a grant from the Social Sciences and Humanities research Council to all other authors. We also want to thank Jean Bégin, statistician in the department of psychology at the Université du Québec à Montréal, for all advices on statistical analyses.

\section{References}

1. American Psychiatric Association (2013) Diagnostic and statistical manual of mental disorders, ( $5^{\text {th }}$ edn) Washington, DC.

2. Arntz A, Tiesema M, Kindt M (2007) Treatment of PTSD: A comparison of imaginal exposure with and without imagery rescripting. J Behav Ther Exp Psychiatry 38: 345-370.

3. Bigelow DA, Brodsky G, Stewart L, Olson M (1982) The concept and measurement of quality of life as a dependent variable in evaluation of mental health services.

4. Blake DD, Weathers FW, Nagy LM, Kaloupek DG, Gusman FD, et al. (1995) The development of a clinician-administered PTSD Scale. J Trauma Stress 8 : 75-90.

5. Bolton E, Holohan DR, King LA, Wing DW (2004) Acute and post-traumatic stress disorder. In: Thomas JC, Hersen M (eds.) Psychopathology in the workplace: Recognition and adaptation. Brunner-Routledge, New York.

6. Crits-Christoph P, Gibbons MBC, Ring-Kurtz S, Gallop R, Stirman S, et al (2008) Changes in positive quality of life over the course of psychotherapy. Psychotherapy: Theory, Research, Practice, Training 45: 419-430.

7. Cronbach LJ (1951) Coefficient alpha and the internal structure of tests. Psychometrika 16: 297-334.

8. Defraia GS (2013) Organizational outcomes following traumatic workplace incidents: A practice-based exploration of impact of incident severity level. Social Work in Mental Health 11: 404-433.

9. First MB, Spitzer RL, Gibbon M, Williams JBW (1996) Structured clinica interview for axis I DSM-IV disorders-patient edition. NY State Psychiatric, New York.

10. Foa EB, Keane TM, Friedman MJ, Cohen JA (2008) Effective treatments for PTSD: Practice guidelines from the International Society for Traumatic Stress studies. Guilford Press, New York.

11. Freeston MH (1997) Développement et validation de l'Échelle de Fonctionnement psychosocialMontréal, Canada

12. Germain V, Marchand A, Bouchard S, Drouin MS, Guay S (2009) Effectiveness of cognitive behavioural therapy administered by videoconference for posttraumatic stress disorder. Cogn Behav Ther 38: 42-53.

13. Gladis MM, Gosch EA, Dishuk NM, Crits-Christoph P (1999) Quality of life: Expanding the scope of clinical significance. J Consult Clin Psychol 67: 320-331.

14. Grunert BK, Matloub HS, Sanger JR, Yousif NJ (1990) Treatment of posttraumatic stress disorder after work-related hand trauma. J Hand Surg 15: 511-515.

15. Hofmann SG, Wu JQ, Boettcher $H$ (2014) Effect of cognitive-behavioral therapy for anxiety disorders on quality of life: A meta-analysis. J Consult Clin Psychol 82: $375-391$.

16. Jonas DE, Cusack K, Forneris CA, Wilkins TM, Sonis J, et al. (2014) Psychological and pharmacological treatments for adults with posttraumatic stress disorder (PTSD). Agency for Healthcare Research and Quality, Rockville, MD.

17. Khoo A, Dent MT, Oei TP (2011) Group cognitive behaviour therapy for military service-related post-traumatic stress disorder: Effectiveness, sustainability and repeatability. Aust N Z J Psychiatry 45: 663-672. 
Citation: Sophie L, Dominic BP, Stéphane G, Geneviève B, André M (2017) Evolution of Posttraumatic Stress Symptoms and Quality of Life throughout a Cognitive Behavioral Treatment for Workplace Trauma Victims. J Ment Disord Treat 3: 137. doi:10.4172/2471-271X.1000137

18. Lacerte S, Bruyninx S, Bourdon DÉ, Marchand A (2015) Quality of life in workplace trauma victims with posttraumatic stress disorder: A systematic review. Manuscript submitted for publication.

19. Lanctôt N, Guay S (2014) The aftermath of workplace violence among healthcare workers: A systematic literature review of the consequences. Aggress Violent Behav 19: 492-501.

20. Laurenceau JP, Hayes AM, Feldman GC (2007) Some methodological and statistical issues in the study of change processes in psychotherapy. Clinical Psychology Review 27: 682-695.

21. Lerner D, Amick III BC, Rogers WH, Malspeis S, Bungay K, et al. (2001) The work limitations questionnaire. Medical Care 39: 72-85.

22. Lutz W, Lowry J, Kopta SM, Einstein DA, Howard KI (2001) Prediction of doseresponse relations based on patient characteristics. J Consult Clin Psychol 57: 889-900.

23. Martel J, Dupuis G (2006) Quality of work life: Theoretical and methodological problems, and presentation of a new model and measuring instrument. Social Indicators Research 77: 333-368.

24. Muschalla B, Linden M (2013) Different workplace-related strains and different workplace-related anxieties in different professions. J Occup Env Med 55: 978-982.

25. Olatunji BO, Cisler JM, Tolin DF (2007) Quality of life in the anxiety disorders: A meta-analytic review. Clin Psychol Rev 27: 572-581.

26. Pacella ML, Hruska B, Delahanty DL (2013) The physical health consequences of PTSD and PTSD symptoms: A meta-analytic review. J Anxiety Disord 27: 33-46.
27. Papa A, Follette WC (2015) Dismantling studies of psychotherapy. The Encyclopedia of Clinical Psychology: 1-6.

28. Rosen GM, Grunert BK (2012) Posttraumatic stress disorder in the workplace. In: Bush SS, Iverson GL (eds.) Neuropsychological assessment of work-related injuries Guilford Press, New York, pp: 163-186.

29. Schnurr PP, Hayes AF, Lunney CA, McFall M, Uddo M (2006) Longitudinal analysis of the relationship between symptoms and quality of life in veterans treated for posttraumatic stress disorder. J Consult Clin Psychol 74: 707-713.

30. Schnurr PP, Lunney CA (2012) Work-related outcomes among female veterans and service members after treatment of posttraumatic stress disorder. Psychiatric Services 63 : 1072-1079.

31. Sijbrandij M, Engelhard IM, de Vries GJ, Luitse JS, Carlier IV, et al. (2013) The role of injury and trauma-related variables in the onset and course of symptoms of posttraumatic stress disorder. J Clin Psychol Med Settings 20: 449-455.

32. Stergiopoulos E, Cimo A, Cheng C, Bonato S, Dewa CS (2011) Interventions to improve work outcomes in work-related PTSD: A systematic review. BMC Public Health 11: 838.

33. WHOQOL Group (1995) The World Health Organization Quality of life assessment (WHOQOL): Position paper from the World Health Organization. Soc Sci Med 41: 1403-1409.

34. WHOQOL Group (1998) Development of the World Health Organization WHOQOL-BREF quality of life asessment. Psychol Med 28: 551-558. 